(2) Open Access Full Text Article

REVIEW

\title{
Primary infragenicular angioplasty for diabetic neuroischemic foot ulcers following the angiosome distribution: a new paradigm for the vascular interventionist?
}

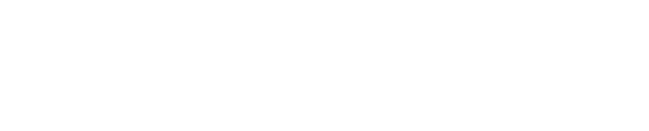

\author{
Vlad Alexandrescu' \\ Gerard Hubermont ${ }^{2}$ \\ 'Department of Vascular Surgery, \\ Princess Paola Hospital, Marche- \\ en-Famenne, Belgium; ${ }^{2}$ Department \\ of Diabetology, Princess Paola \\ Hospital, Marche-en-Famenne and \\ Sainte-Thérèse Hospital, Bastogne, \\ Belgium
}

\begin{abstract}
The angiosome principle was first described by Jan Taylor in 1987 in the plastic reconstructive surgery field, providing useful information on the vascular anatomy of the human body. Specifically concerning foot and ankle pathology, it may help the clinician to select better vascular access and specific strategies for revascularization. This knowledge may be particularly beneficial when treating diabetic neuroischemic foot wounds associated with particularly aggressive atherosclerotic disease and a poor collateral circulation. The implementation of angiosome-based strategies in diabetic infragenicular vascular reconstruction may afford encouraging wound healing and limb preservation rates using both bypass and endovascular techniques. The minimal invasiveness of these novel strategies enables us to perform more specific and more distal tibial and/or foot arterial reconstructions, in one or multiple targeted vessels. This paper reviews the available literature on this revascularization strategy and focuses on the potential benefit of angiosome-guided primary angioplasty for diabetic ischemic foot ulcers.
\end{abstract}

Keywords: critical limb ischemia, diabetic foot, limb salvage, angiosomes, angioplasty

\section{Introduction}

Although surgical bypass still plays a key role in revascularization of critical limb ischemia (CLI), ${ }^{1}$ increasing clinical experience over the past two decades shows encouraging results for primary endovascular strategies, with acceptable feasibility, low complication rates, ${ }^{2-4}$ and limb salvage rates comparable with surgery. ${ }^{2-5}$ Diabetes mellitus is becoming increasingly common in CLI presentations featuring prolonged foot inflammation or tissue necrosis. This currently involves frail patients at high perioperative risk and affected by numerous vascular comorbidities. ${ }^{1,2}$ This specific patient population, with systemic atherosclerosis, neuroischemic limb ulcers, gangrene, and sepsis (the so-called "diabetic foot syndrome") is prone to a higher rate of periprocedural surgical complications. ${ }^{1,2,5}$ Therefore, endovascular techniques may have many applications in this field ${ }^{2}$ because of their low invasiveness, absence of scarring, and lack of need for venous conduits. ${ }^{2,6,7}$ These strategies seem to have the advantages of enabling simultaneous multiple vessel recanalization with high reproducibility if necessary, ${ }^{6,7}$ resulting in shorter hospital stays and health care expenditure. $^{1-7}$
Correspondence: Vlad Alexandrescu Department of Vascular Surgery, Princess Paola Hospital, Rue du Vivier 21, 6900 Marche-en-Famenne, Belgium Tel +32 842I 9I I I

$\mathrm{Fax}+32$ 843I 66I3

Email v.alex@skynet.be 


\section{Common revascularization issues in diabetic patients}

The diabetic ischemic foot, because of its multifaceted pathology, presents well recognized challenges for the vascular interventionist. Chronically and critically oxygendeprived tissues in these frail patients often develop ulcers, extended sepsis, and limb edema. Heavily calcified and occluded calf and foot arteries make all types of vascular reconstruction more difficult. ${ }^{1,2,6}$ Not surprisingly, the main infragenicular arterial axis that supplies the distal wound territory is frequently affected by severe atherosclerotic disease. ${ }^{4,8-10}$ Current critical limb ischemia data suggest that bypass using vein grafts achieves $79 \%-90 \%$ limb salvage rates at 1 year, ${ }^{8}$ while below-knee angioplasty affords technically successful recanalization rates of up to $80 \%$, with comparable rates of limb preservation. ${ }^{1,5,8}$ However, these series have a heterogeneous Rutherford grade II-III component and class 4-6 presentation. ${ }^{1,8}$ It is generally accepted for distal bypass and endovascular reconstructions that the outflow vessel based on angiographic details modulates the strategy for vascular reconstruction and enables technical success and good postoperative patency. ${ }^{1,8}$ From this perspective, the aim is to reirrigate the distal ischemic wound territory directly, but is often achieved indirectly via collaterals surrounding the diseased zone. The decision-making process often focuses on the most suitable artery to be reopened or a patent distal vessel to anchor the bypass. ${ }^{1,2,6-8}$

In the last two decades, some authors have proposed complementary topographic orientations in critical limb ischemia revascularization, because the choice of the most appropriate target vessel starts from a clinical point of view, ie, the wound location. ${ }^{9-11}$ They have described particular tissue sectors of the human body depending on specific arterial and venous irrigation sources, named "angiosomes". ${ }^{9-14}$ The distribution of these angiosomes was eventually sought to harmonize already used arterial reconstruction techniques. ${ }^{10-12}$

\section{Angiosomes and limb salvage}

Parallel efforts to decrease diabetic limb losses have unfolded in the field of plastic reconstructive surgery, by promoting the angiosome model of perfusion. ${ }^{9-14}$ This concept is based on the anatomical studies of Taylor et $\mathrm{al}^{9,12}$ and Attinger et al, ${ }^{10,11,13,14}$ who pioneered preferential strategies for surgical access, tissue reconstruction, and amputation, following specific three-dimensional tissue sectors of the body, ie, angiosomes in specific arteriovenous bundles.
Practical implementation of the angiosome concept in daily limb salvage bypass or transcatheter techniques started only a few years ago. Although few results have been published as yet, and mainly in the vascular interventional field, retrospective studies seem to favor this hypothesis. ${ }^{14-17}$ In a retrospective surgical series of 48 patients, Neville et al ${ }^{15}$ found a $91 \%$ healing rate with a $9 \%$ amputation rate in the angiosome-guided group vs a $62 \%$ healing rate and $38 \%$ amputation rate in the nonangiosome-guided group. In 203 consecutive limbs with critical ischemia and ischemic ulcerations undergoing endovascular reconstruction, Iida et $\mathrm{al}^{18}$ reported limb preservation in $86 \%$ of the angiosome-related group compared with $69 \%$ in the nonspecific treatment group. Consistent with these reports, in a similar series of 76 ischemic ulcers treated by both bypass and endovascular therapies, Varela et al ${ }^{19}$ noted significant better results for wound healing (92\% vs $73 \%$ ) and limb salvage (93\% vs $72 \%$ ) in the angiosome-guided cohort of patients. We have previously published our experience in 98 diabetic patients with ischemic foot ulcers treated by angiosome-oriented primary angioplasty, with similarly encouraging results for limb preservation $(91 \%, 88 \%$, and $84 \%$ at 12,24 , and 36 months, respectively) and wound healing $(85 \%, 81 \%$, and $73 \%$ at the same time intervals). ${ }^{17}$

In practical terms, the angiosome concept delineates the human body into three-dimensional tissue blocks irrigated by specific arteriovenous sources. ${ }^{9-12}$ Adjacent angiosomes are interconnected by numerous "choke vessels" that create an integrated compensatory system between different territories. ${ }^{9-14}$ Although not part of everyday practice, this model already has some contemporary clinical applications, including specific myocardial revascularization, selective visceral embolization, characteristic types of covering flaps, ${ }^{9,12}$ incisions or amputations ${ }^{10,14}$ in plastic reconstructive surgery, ${ }^{9-14}$ and, more recently, targeted bypass or endovascular revascularization for limb salvage in critical limb ischemia. ${ }^{11,13-19}$ In the lower limb, five angiosomes were initially described ${ }^{9,12,13}$ in the foot and lower ankle ${ }^{9-11,13}$ emerging from the three main tibial trunks, ie, the anterior and posterior tibial arteries joined to the peroneal vessels.

\section{Schema for foot and lower ankle angiosomes}

Schematically, the distribution of angiosomes ${ }^{12-17}$ in the foot encompasses: the medial calcaneal, medial plantar, and lateral plantar artery angiosomes derived from the posterior tibial artery supplying the entire plantar heel and the medial and lateral plantar surface beyond the toes; the dorsalis pedis 
angiosome, which extends from the anterior tibial artery, supplying the dorsum of the foot and toes, and also upper anterior perimalleolar vascularization; and the lateral calcaneal artery angiosome (optionally adding the lateral perimalleolar artery angiosome) derived from the peroneal artery, covering the lateral and plantar heel and succeeding the anterior peroneal perforating branch, that connects the latter to the anterior tibial territory (Figure 1). Going up to the superior ankle and distal calf zones, other angiosome territories have been identified, including the anterior perforator artery angiosome (from the peroneal artery) and the lateral malleolar angiosomes with the corresponding medial malleolar angiosomes from the anterior-tibial artery., ${ }^{911,12}$ Some of these perimalleolar angiosomes have been additionally considered by recent reports of revascularization procedures..$^{15,16,18} \mathrm{~A}$ few examples of current angiosome-guided endovascular interventions for diabetic neuroischemic foot wounds are shown in Figures 2-9.

The diabetic foot is a preferential application for topographic revascularization. Availability of the angiosome strategy for infragenicular revascularization seems to represent a particular interest in diabetic patients for several reasons. It has been suggested that the neuroischemic diabetic foot is associated with more distal and aggressive atherosclerotic macroangiopathy, as well as functional microcirculatory impairment induced by both neuropathy and local sepsis..$^{20,21}$ In this specific context, featuring multiple blockades of large and medium-sized foot arteries, ${ }^{20} \mathrm{O}$ 'Neal has outlined the concept of diabetic end-artery occlusive disease. ${ }^{21}$ This hypothesis considers critical irrigation to the foot, whereby medium-sized "patchy atherosclerosis" is associated with acute septic thrombosis and loss of small collaterals with surrounding inflammation. ${ }^{21}$ The end-artery occlusive disease theory ${ }^{21}$ probably explains better why irrigation from a few millimeters of skin to the entire diabetic foot or $\operatorname{leg}^{11,16,17,21}$ relies on specific nourishing vessels, although solely hinged to one specific dominant angiosome-dependent artery. ${ }^{9-12}$ Consequently, it might be emphasized that in these subjects, the more distal and specific the revascularization, the higher the probability of re-establishing an adequate blood supply in a specific amount of threatened tissue.
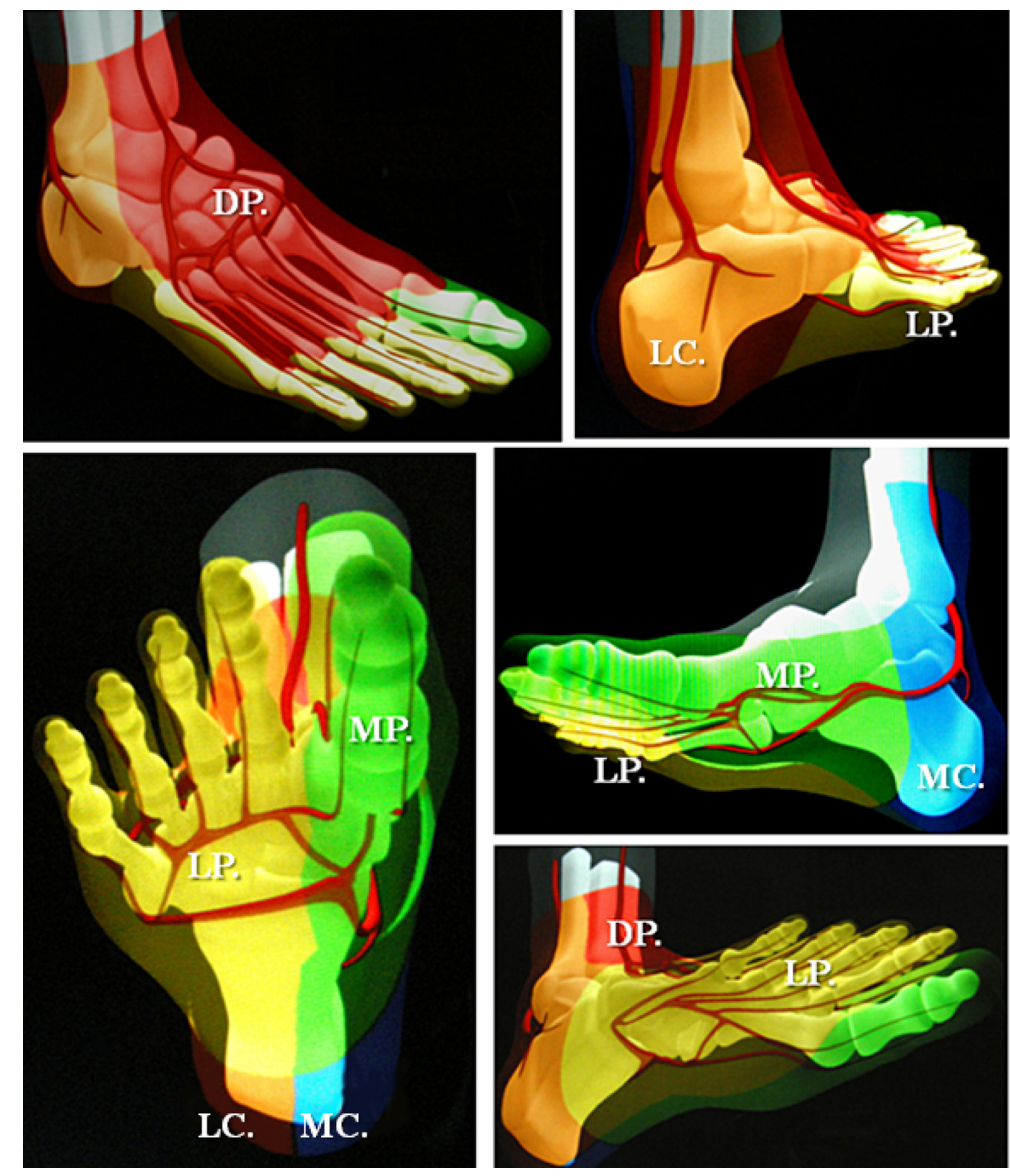

Figure I A simplified illustration of previously suggested angiosomes of the foot and lower ankle.

Abbreviations: DP, dorsalis pedis artery angiosome; LP, lateral plantar artery angiosome; MP, medial plantar artery angiosome; LC, lateral calcaneal artery angiosome; MC, medial calcaneal artery angiosome. 


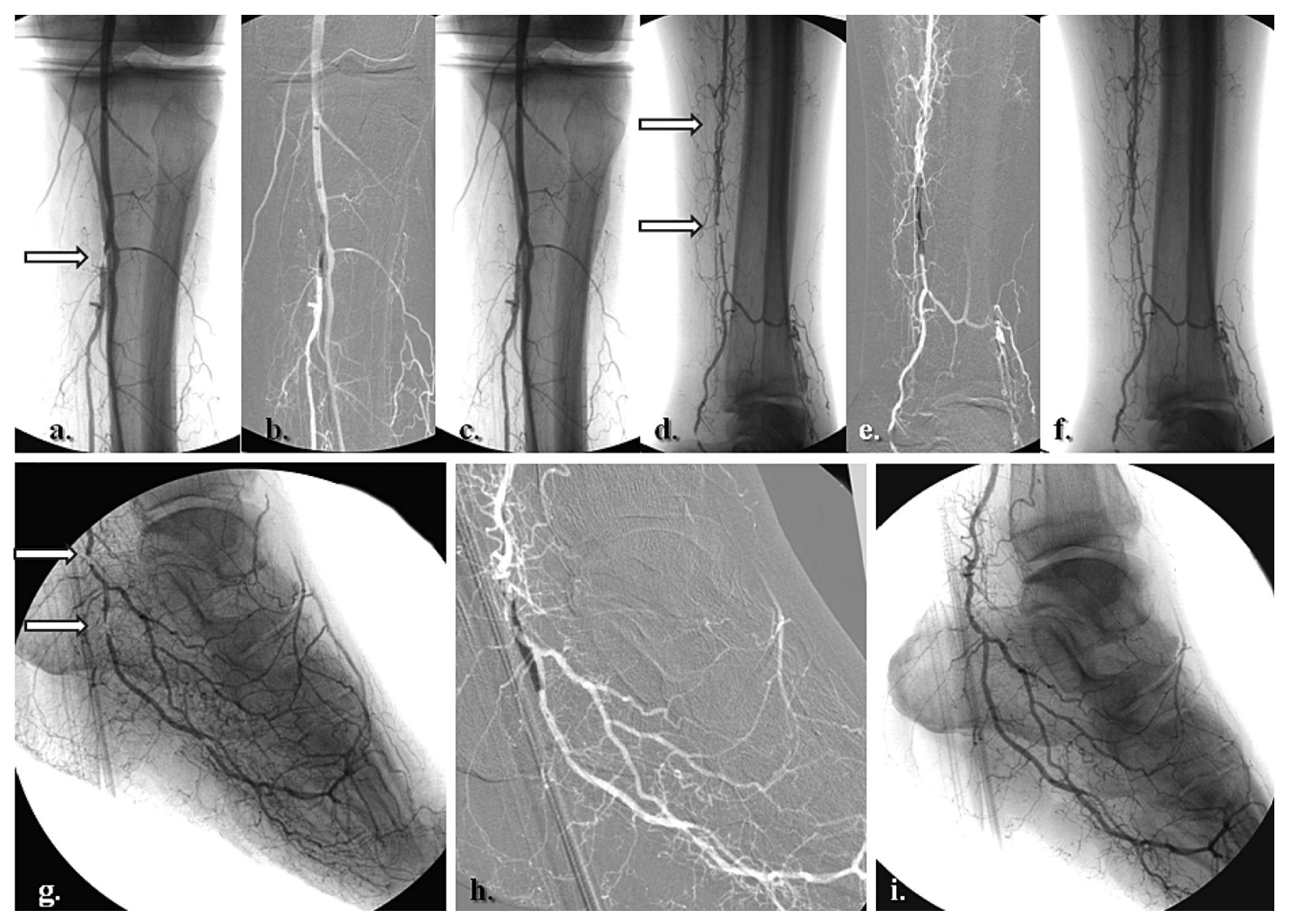

Figure 2 Selective revascularization of the posterior tibial and lateral plantar artery angiosome: $(\mathbf{a}-\mathbf{f})$ Staged angioplasties in the posterior tibial artery. ( $(\mathbf{g}-\mathbf{i})$ Selective angioplasties in the lateral plantar artery and its appended angiosome.
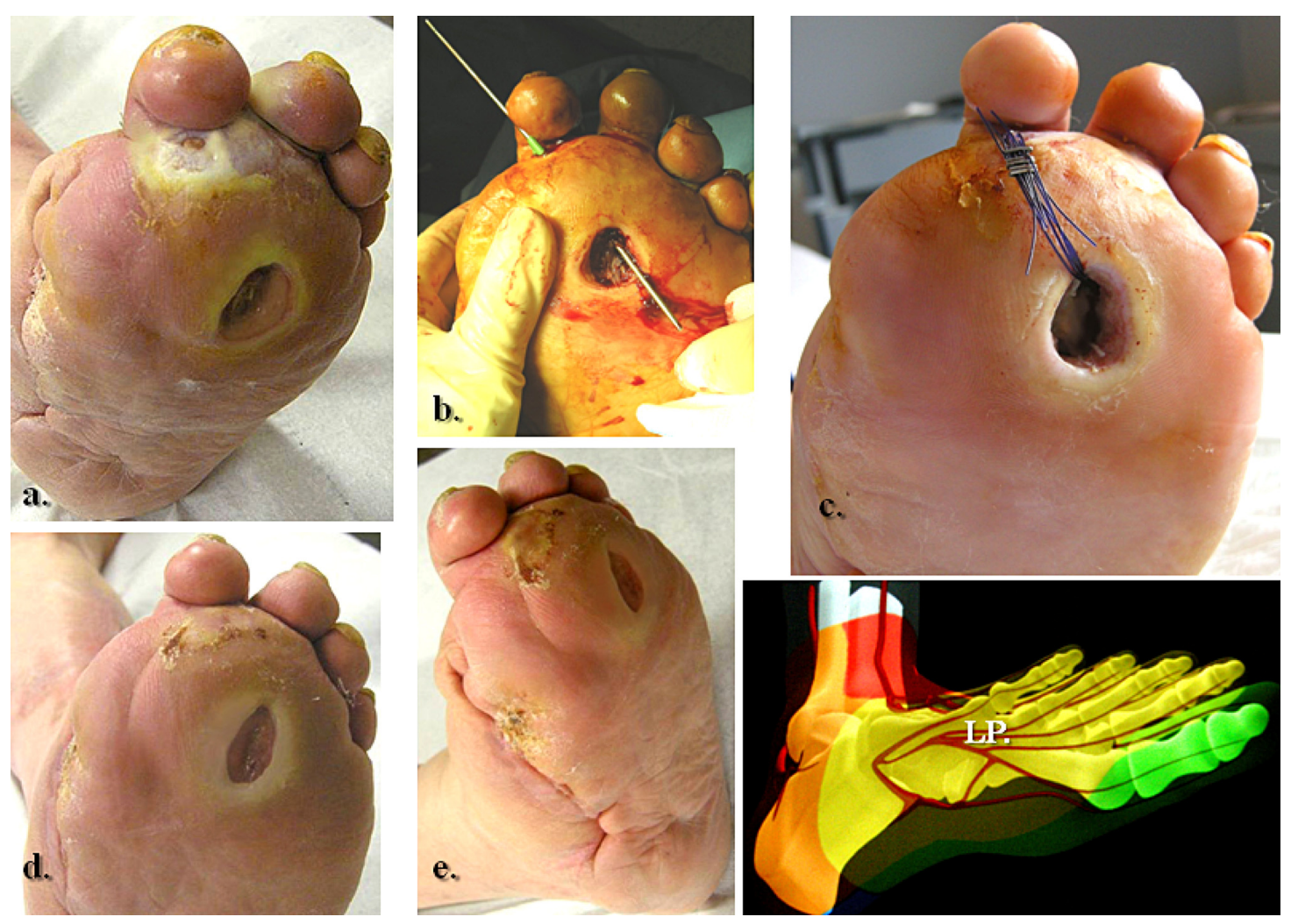

c.

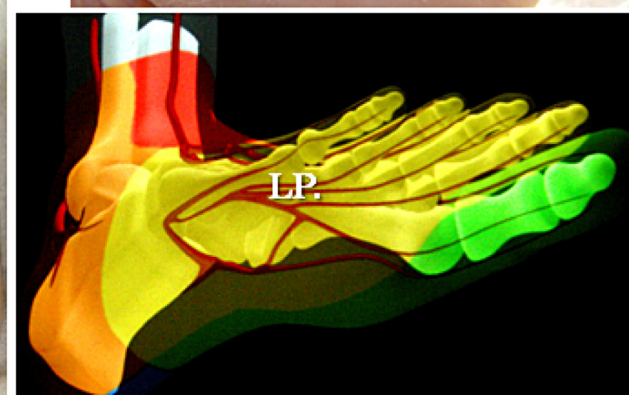

Figure 3 Clinical correspondence of the angiographic pattern showed in Figure 2. A neuroischemic plantar ulcer in an acute diabetic foot presentation: (a) Initial clinical aspect featuring a lateral plantar artery hypoperfusion and sole forefoot abscess. (b) and (c) Abscess drainage and debridement. (d) and (e) Clinical evolution at weeks 3 and 5. 


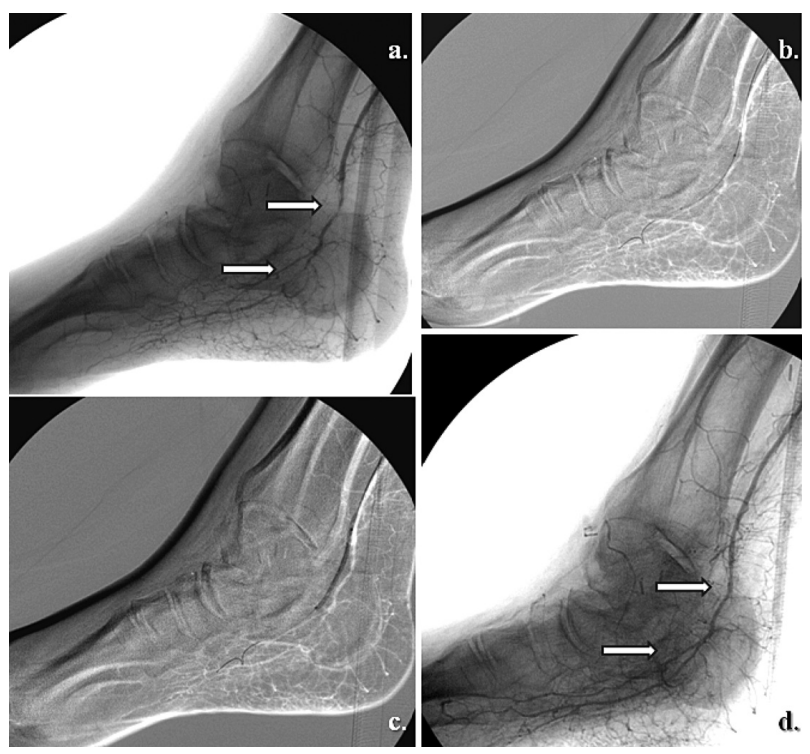

Figure 4 Selective revascularization of the distal posterior tibial artery and its appended medial plantar artery angiosome. (a) and (b) Selective angioplasty in the posterior tibial artery, and (c) and (d) specific revascularization of the medial plantar artery and its angiosome.

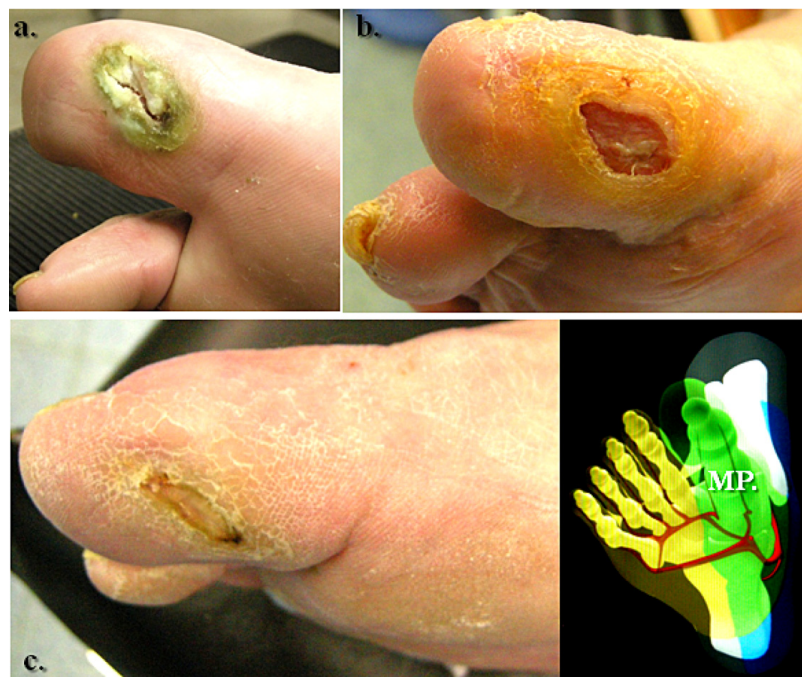

Figure $5 \mathrm{~A}$ right hallux neuroischemic sole ulceration matching the angiographic features showed in Figure 4. There is medial plantar artery hypoperfusion in an end-artery occlusive disease pattern for the first toe. (a) Initial presentation and (b) clinical evolution at I month after angioplasty. (c) Results 2 months later.
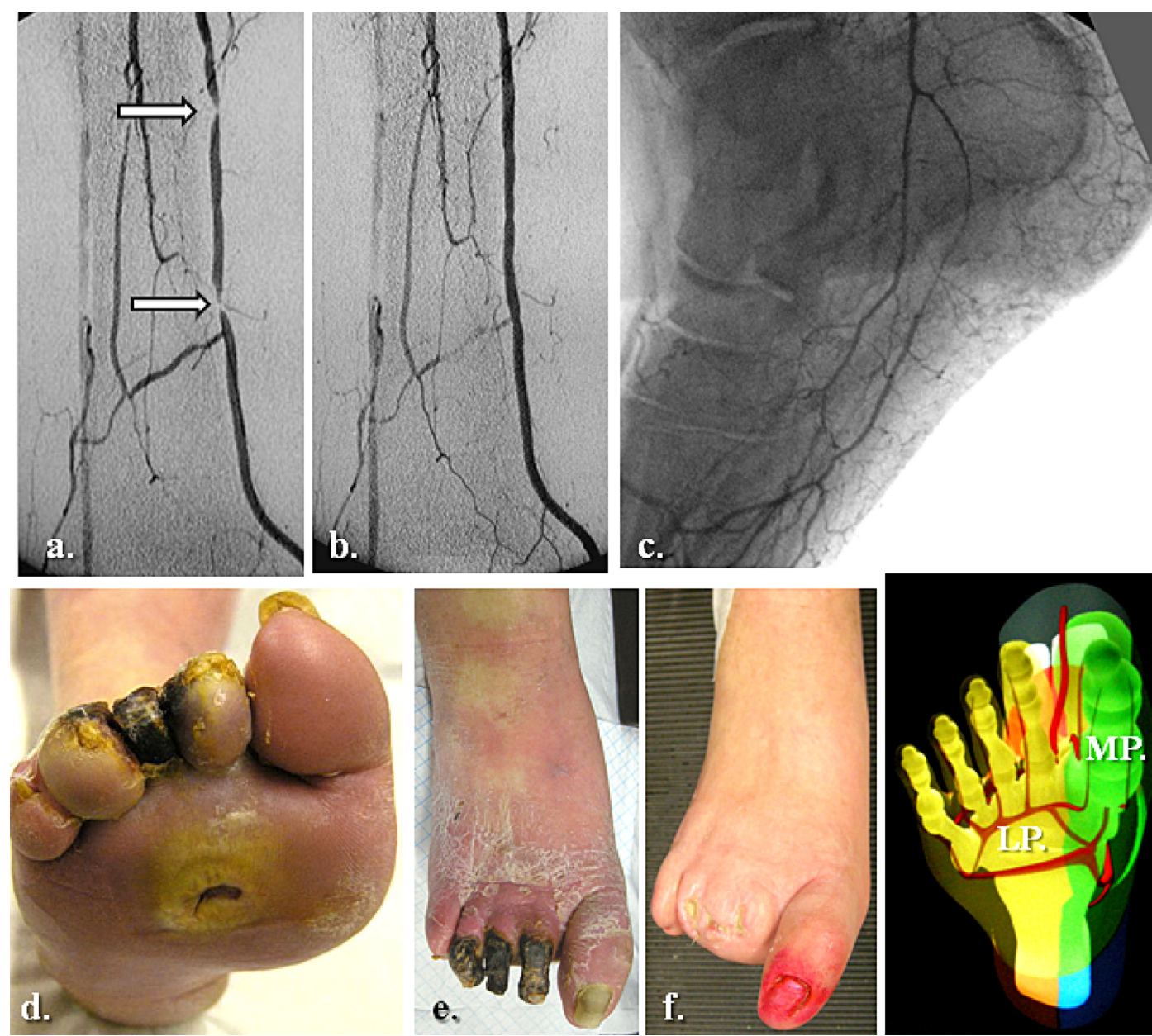

Figure 6 Global medial and lateral plantar artery critical ischemia and acute diabetic foot syndrome matching staged occlusions in the posterior tibial artery (with end-artery occlusive disease model to the sole). (a) Prime posterior tibial artery staged subocclusive lesions. (b) and (c) The reestablished flow in the posterior tibial and both right plantar arteries. (d) The initial clinical aspect. (e) Subsequent evolution at 3 weeks. (f) Clinical results after 5 months of team surveillance. 

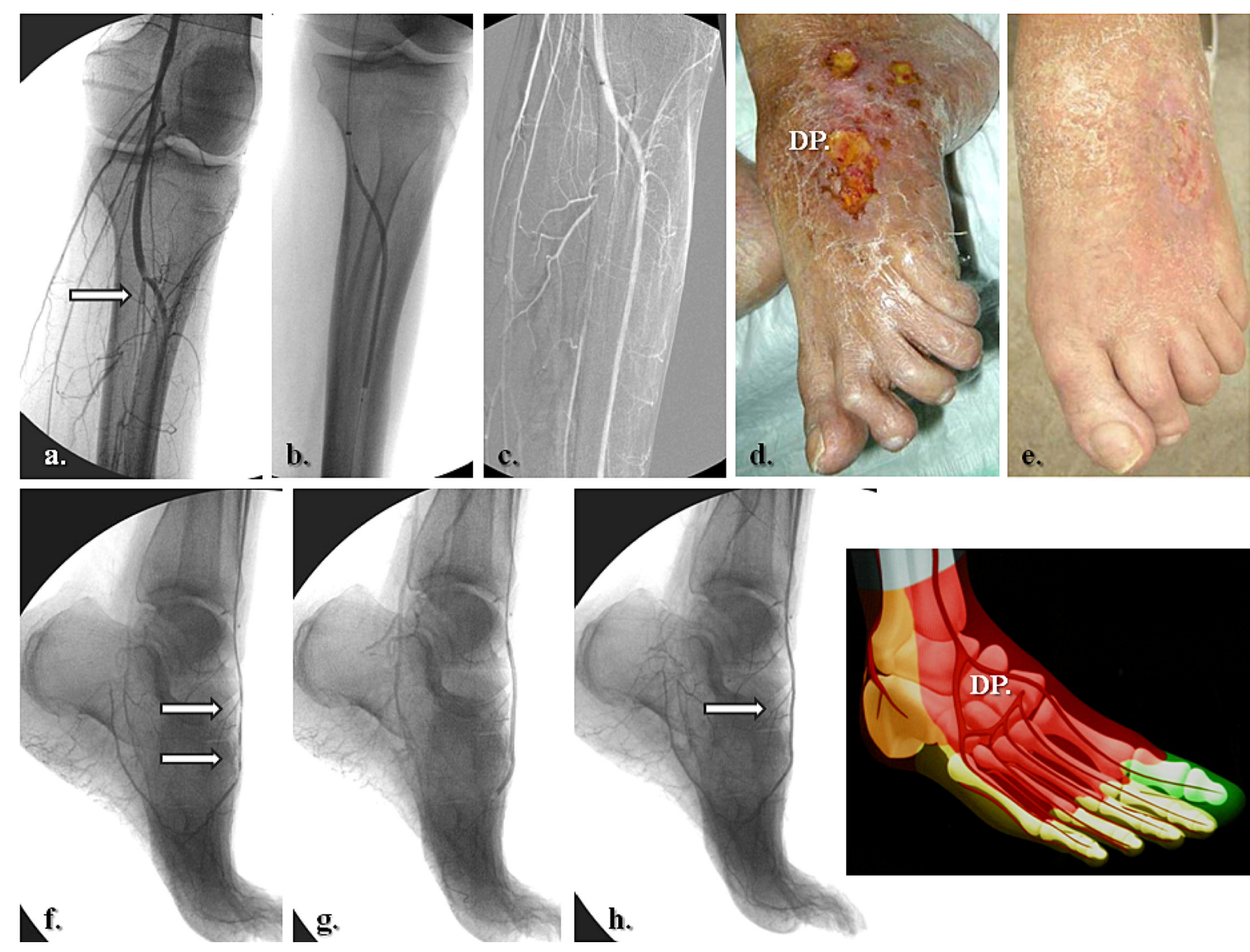

Figure 7 Selective anterior tibial and related dorsalis pedis artery angiosome: (a-c) Primary staged anterior tibial angioplasty, (d) initial dorsal foot ulceration, (e) clinical results at I month following $(\mathbf{f}-\mathbf{h})$ associated dorsalis pedis selective angioplasty.

The association between the end-artery occlusive disease theory ${ }^{21}$ and the broader angiosome concept may reveal promising results in diabetic wound regeneration, ${ }^{13,15-19,22}$ particularly if specific pedal and/or plantar revascularizations are added to those of the tibial trunk ${ }^{14,17,18,23}$ in single interventions. This also underscores the advantage of endovascular strategies to allow multiple below-knee and below-ankle vessel reconstructions, in addition to surgery. On the complex background of a diabetic foot, the angiosome concept may equally allow vascular interventionists to select more specific wound-oriented vascular reconstruction with probably a better prognosis in terms of tissue healing, as suggested in recent reports by Setacci et al, ${ }^{16}$ Clemens and Attinger, ${ }^{22}$ and Bazan. ${ }^{24}$ Beyond the initial clinical orientation (focusing on the specific location of foot ulcers, Figures 1-9), other laboratory examinations may help the clinician to choose the appropriate angiosome for revascularization, ie, Doppler assessment of the dominant foot arch, ${ }^{11-13}$ angiographic computed tomographic or magnetic resonance evaluation of homolateral and contralateral limb arterial patterns, ${ }^{17}$ and transcutaneous oxygen pressure monitoring stratification in adjacent angiosomes. ${ }^{17}$

\section{Challenging endovascular procedures in diabetic infragenicular trunks}

Figures 2, 4, 7, and 9 show the main categories of targeted angiosome revascularization and indicate the presence of specific calcifications in the tibial, dorsal foot, or plantar diabetic arteries. These calcific deposits represent one of the major technical concerns for the vascular interventionist. ${ }^{2,6,17}$ Inasmuch as nondiabetic subjects seem to develop intimal, eccentric, and patchy arterial wall calcific deposits (ie, type I calcifications), ${ }^{25}$ diabetic infrapopliteal atherosclerosis affects the medial layer, with mostly concentric continuous wall calcifications ( "Mönckeberg sclerosis" or type II calcification). ${ }^{25}$ Although the precise etiology is unknown, their severity and spread have been related to the duration 


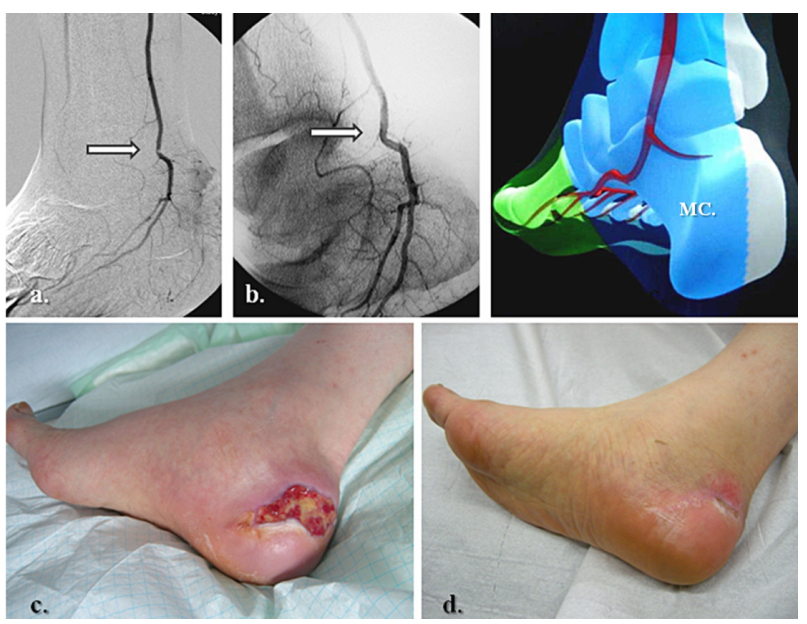

Figure 8 Specific posterior tibial and adjacent medial calcaneal artery angiosome revascularization in heel related wound. (a) Tight stenosis in the distal posterior tibial artery, above emergence of the medial calcaneal branch, (b) Angiographic result after selective angioplasty, (c) initial presentation of heel ulcer, and (d) clinical results after 5 weeks of team surveillance.

of diabetes, concomitant autonomic neuropathy, and specific alterations in the vasa vasorum. ${ }^{10,25}$ Type II calcifications undoubtedly present challenges for revascularization using endoluminal or surgical techniques in these rigid conduits with

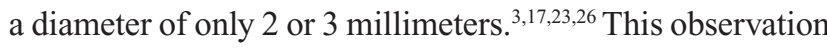
becomes even more manifest when choosing an angiosome orientation for tibial revascularization, ${ }^{17,19,26}$ acknowledging that the appropriate angiosome-dependent artery may not necessarily be the simplest vessel to treat. ${ }^{15-17}$ It has been shown that severely ischemic territories with tissue loss more often include long segments of chronic and calcific arterial occlusions in the corresponding angiosome-related vessels. ${ }^{16-19}$

Another challenging factor to be faced by the vascular specialist when planning treatment of a diabetic ulcer is the control of local neuropathy. Beyond chronic exposure of the foot with sensory loss to microtrauma, skin tears, and deformation, neuropathy adds characteristic functional microcirculatory impairment ${ }^{16}$ by autonomic denervation. ${ }^{16,20,21}$ The latter strongly affects residual perfusion of the skin by capillary steal, impairing the local healing process ${ }^{16,21}$ and consequently the distal arterial runoff to the foot (an important consideration in any vascular reconstruction). ${ }^{16,20}$ An additional element of the diabetic environment is local sepsis. This component of the diabetic foot puzzle commonly causes purulent collections, swelling, and adjacent hyperpressure in the form of foot compartment syndromes. ${ }^{20,21}$ A consequent neural (peripheral entrapment) and remote arterial collateral depletion has been described. ${ }^{20,21,26}$ Therefore, the compensatory capillary network of angiosomes (ie, "choke" vessels) 9-14,16,26 seems strongly determined by the duration of diabetes and

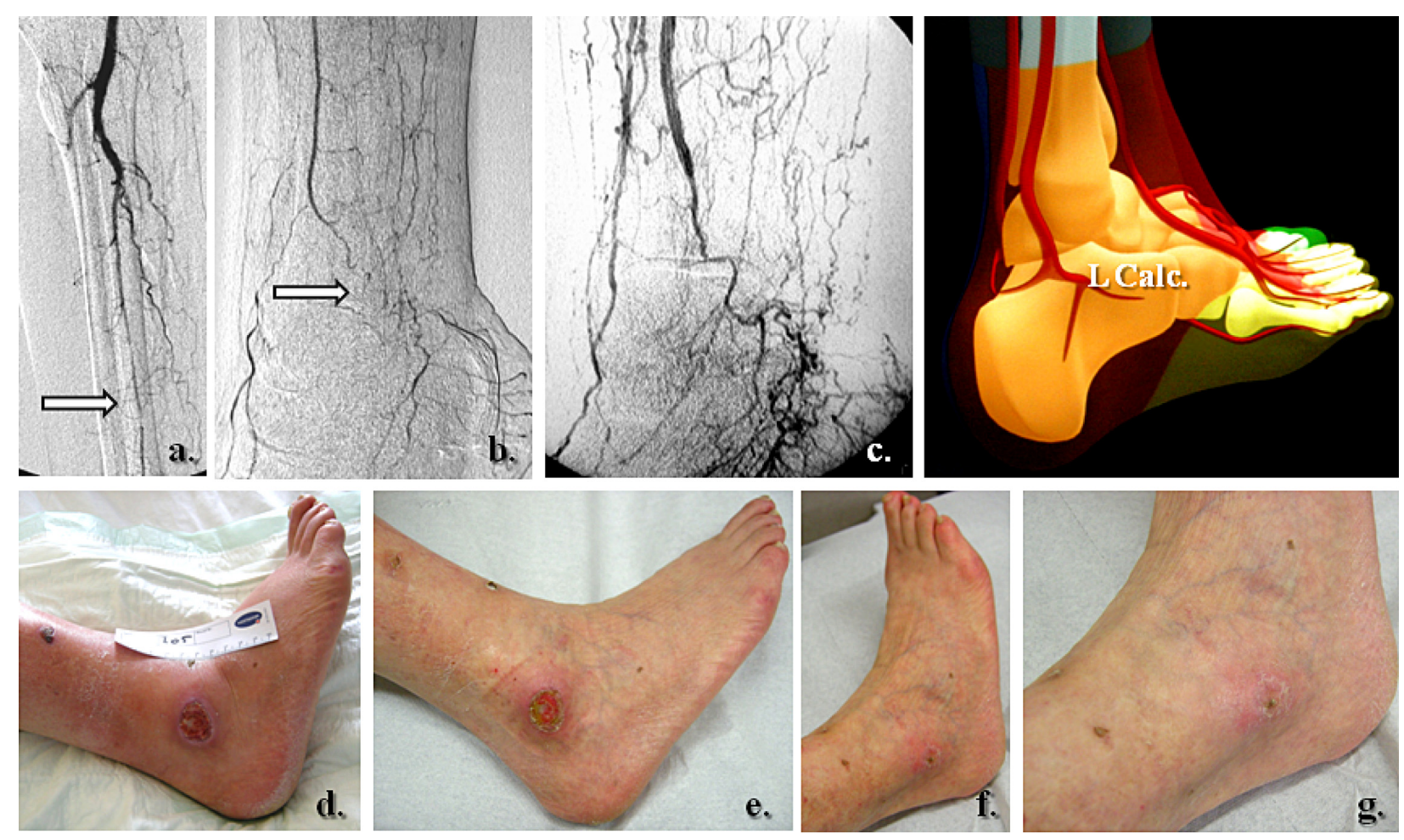

Figure 9 Lateral calcaneal artery and peroneal main flow-related angiosome ulceration. (a, b) Initial pattern of perfusion featuring the peroneal artery as single and severely diseased (end-artery occlusive model) calf vessel, (c) re-established flow in the peroneal territory, (d) prime aspect of lateral calcaneal and inframalleolar tissue defect, and (e-g) subsequent clinical evolution at weeks I, 5, and 6. 
chronic inflammation, ${ }^{20,21,26}$ indicating the need to treat ischemic foot areas by more distal and more specific vascular reconstructions. ${ }^{15-17}$ These specific diabetic foot challenges seem to increase the postoperative risk in terms of tissue recovery ${ }^{6,14,16,17,20,26}$ which may still jeopardize limb salvage after successful revascularization..$^{14,16,21,26}$ Because the angiosome strategy seems to be advantageous when performing revascularization, ${ }^{14-16,18,24}$ with particular utility in the diabetic foot ischemic model, ${ }^{13,17,22}$ it seems reasonable to predict an increasing role for this concept $\mathrm{t}^{16,17,19,24}$ in an era of expanding endovascular applications, including the "first approach"26-29 and hybrid surgical and transcatheter procedures. ${ }^{27,28}$

\section{Limitations and challenges of angiosome-based strategies}

Our group has encountered two main challenges while using the angiosome strategy for infragenicular angioplasty. First, the angiosome concept of revascularization has shifted our indications from "which vessel is most suitable for revascularization" to a multidisciplinary clinical consideration of "which region of perfusion governed by which artery should be treated?" This policy has engaged our vascular group in more laborious procedures, acknowledging that the most suitable angiosome-dependent artery to treat may not necessarily be the simplest vessel to recanalize. ${ }^{15-19}$ As emphasized earlier, extended calcifications in long segment chronic occlusions or tight stenoses are commonly encountered during angiosometargeted revascularization. Following local learning curves and evolving skills, our initially observed global technical failure rate of $20 \%{ }^{17}$ seems to match similar critical limb ischemia angioplasty feasibility reports, ranging from $69 \%-85 \%$ for occlusions and 77\%-95\% for severe stenoses. ${ }^{3-7,30,31}$ In an attempt to address these technical limitations, we have favored antegrade approaches, long $(50-70 \mathrm{~cm})$ sheaths reinforced by stiff catheters (Lumax/Cook, UK or 5-French Pier, Cordis, New York, NY) and a stiff shaft with 0.018 in over-a-wire balloons (ReeKross, Clear Stream Technologies Ltd, Wexford, Ireland). Occasionally, in heavily calcified vessels, cutting balloons (Boston Inc, Fall River, MA) or blunt microdissection devices (Frontrunner, Cordis) have been used to improve access (Figures 2 and 3). New endovascular techniques (eg, tibial retrograde approaches) or recanalization devices based on rotational endarterectomy, excimen laser, or ultrasound debulking therapy, have seldom been used by our team, but may offer promise for mandatory topographic recanalization. . $3,26,31,33$

The second limitation relates to occasional anatomical variation of the main angiosome boundaries between patients.
Although constant in number, ${ }^{10-14}$ each of the main arterial bundles in the ischemic foot and ankle remains strongly dependent on the collateral supply. ${ }^{10-13,18,19}$ The end-artery occlusive disease theory ${ }^{21}$ referred to earlier emphasizes the deficiency of medium-sized and small-sized collateral networks ${ }^{9,14}$ in the diabetic foot syndrome, the angiosome equivalents of which being the choke vessels. Unlikely nondiabetic critical limb ischemia presentations, diabetic neuroischemic wound territories show major collateral deterioration, ${ }^{20-22,32}$ with no opportunity for a compensatory collateral circulation. ${ }^{21,32}$ The amount of tissue depending on one specific arterial source to be reopened varies from a few square millimeters of skin, to the entire foot or leg. ${ }^{21}$ In our experience, in presentations showing complex overlapping wounds with adjacent angiosomes with questionable collateral resources, we orient procedures toward tandem or multiple angiosome reopening, if technically feasible.

\section{Recent reports of infragenicular- targeted revascularizations}

In a recent review of our group experience based on multidisciplinary follow-up, we compared the efficacy of below-knee angioplasty in two consecutive cohorts of diabetic patients with and without the angiosome concept. ${ }^{32}$ Although not reaching statistical significance for primary $(P=0.813)$ and secondary patency $(P=0.511)$, we found a significant difference in wound healing (hazards ratio [HR] 2.19, $P=0.025$ ) and limb preservation (HR: 2.32, $P=0.035$ ) between these consecutive groups, with better results for angiosome-guided revascularization. ${ }^{32}$ In the same retrospective analysis, ${ }^{32}$ although the number of initial technical failures in the angiosome-guided primary angioplasties was marginally higher ( $21 \%$ vs $18 \%$ for more challenging lesions), we observed no significant difference $(P<0.05)$ in terms of need for reintervention or perioperative morbidity and mortality between angiosome-oriented vs nonangiosome-oriented angioplasties. ${ }^{32}$ Other published reports have found parallel healing/nonhealing and limb salvage rates, ie, $91 \%$ vs $62 \%{ }^{15}$ compared with $85 \%$ vs $76 \%$ in our group ${ }^{32}$ and $86 \%$ vs $69 \%,{ }^{18}$ analogous to $90 \%$ vs $84 \%$ in our registry data. ${ }^{32}$ In summary, there has been equal benefit for both surgery and endovascular strategies using angiosome-guided approaches. ${ }^{15,19,32}$

The BASIL (Bypass versus Angioplasty in Severe Ischaemia of the Leg) trial ${ }^{5}$ reports similar early amputation-free survival rates for surgery and endovascular techniques in nontopographic below-knee revascularizations, although less than one third of patients had diabetes. The same prospective study did not detect a significant decrease in survival rates for 
diabetic subjects using both therapeutic options. ${ }^{5,31}$ A recent analysis conducted by $\mathrm{O}^{\prime}$ Brien-Irr et $\mathrm{a}^{33}$ studied the clinical outcome of 106 cases divided in Rutherford category 4 and 5 chronic limb ischemia presentations and following infrainguinal endovascular treatment, comprising 32\% and $58 \%$ of diabetic patients, respectively. The authors found that for Rutherford category 5 cases, comparable limb salvage rates were $82 \%$, with early wound healing occurring in only $21 \%$ of cases without target extremity revascularization. They concluded that refining patient selection and clinical indications may substantially improve the outcome of percutaneous transluminal angioplasty. ${ }^{33}$ Hoping to provide more accurate information upon the tibial runoff and the collateral reserve in each critical limb ischemia pattern of presentation, Mustapha et $\mathrm{al}^{34}$ recently proposed an original classification and interventional protocol for AM-guided below-knee interventions. They graded from 0-3 different types of ischemic crural patterns, adding a three-level collateral scoring system that may help the interventionist to better choose the target artery and consequent chronic total occlusion (CTO) strategy to be deployed. ${ }^{34}$

\section{Angiosome concept as a component of diabetic team work}

Contemporary clinical experience suggests that using the aforementioned new technologies and principles of treatment, like the angiosome concept, a linear correlation between successful revascularization and unreserved tissue healing is difficult to ascertain $^{20,26,27}$ unless appropriate multidisciplinary surveillance is undertaken. This seems particularly true for diabetic neuroischemic foot wounds. ${ }^{14,16,17,20,22,26-29}$ It is also generally accepted that people with diabetes should be offered specific education about preventive foot care. ${ }^{20,29,35}$ The effectiveness of any educational program is critically linked to the availability of complementary clinical services. ${ }^{36}$ Trying to merge primary (the patient) with secondary (the medical team) prevention in the diabetic foot group at our institution, we have included patients and their general practitioners as members of the team, with their active participation in all therapeutic decisions.

\section{Conclusion}

Implementation of angiosome-based strategies in infragenicular interventions may improve wound healing and limb preservation rates using bypass and endovascular techniques. It may also offer the opportunity in primary angioplasty to orient pedal/plantar revascularizations more specifically and more distally. Incorporating the angiosome model in contemporary below-knee team strategies for revascularization might be useful, although further comparative and prospective data are needed to evaluate this concept fully.

\section{Acknowledgments}

We would like to acknowledge the members of our institutional radiology, endocrine, and diabetic foot teams for their support in generating the data and figures included in this paper.

\section{Disclosure}

The authors report no conflicts of interest in this work.

\section{References}

1. Norgreen L, Hiatt WR, Dormandy JA, et al. Inter-Society Consensus for the management of peripheral arterial disease (TASC II). Eur J Vasc Endovasc Surg. 2007;33 Suppl 1:S32-S55.

2. Blevins WA, Schneider PA. Endovascular management of critical limb ischemia. Eur J Vasc Endovasc Surg. 2010;39:756-761.

3. Conrad MF, Kang J, Cambria RP, et al. Infrapopliteal balloon angioplasty for the treatment of chronic occlusive disease. J Vasc Surg. 2009;50:799-805.

4. Romiti M, Albers M, Brochado-Neto FC, et al. Meta-analysis of infrapopliteal angioplasty for chronic critical limb ischemia. $J$ Vasc Surg. 2008;47:975-981.

5. Adam DJ, Beard JD, Cleveland T, et al; BASIL trial participants. Bypass versus angioplasty in severe ischemia of the leg (BASIL): Multicentre, randomized controlled trial. Lancet. 2005;366:1925-1934.

6. Markose G, Bolia A. Below the knee angioplasty among diabetic patients. J Cardiovasc Surg (Torino). 2009;50:323-329.

7. Faglia E, Mantero M, Caminiti M, et al. Extensive use of peripheral angioplasty, particularly infrapopliteal, in the treatment of ischemic diabetic foot ulcers: Clinical results of a multicentric study of 221 consecutive diabetic subjects. J Intern Med. 2002;252:225-232.

8. Simms M. Peripheral vascular disease and reconstruction. In: The Foot in Diabetes. 4th ed. Chichester, UK: J Wiley and Sons Ltd: 2007.

9. Taylor GI, Palmer JH. The vascular territories (angiosomes) of the body: Experimental studies and clinical applications. Br J Plast Surg. 1987;40:113-141.

10. Attinger CE, Cooper $P$, Blume $P$, et al. The safest surgical incision and amputations applying the angiosomes principle and using the Doppler to assess the arterial-arterial connections of the foot and ankle. Foot Ankle Clin North Am. 2001;6:745-801.

11. Attinger CE, Evans KK, Bulan E, et al. Angiosomes of the foot and ankle and clinical implications for limb salvage: Reconstruction, incisions and revascularization. Plast Reconstr Surg. 2006;117 (7 Suppl):261S-293S.

12. Taylor GI, Pan WR. Angiosomes of the leg: Anatomic study and clinical implications. Plast Reconstr Surg. 1997;4:183-198.

13. Attinger CE, Evans KK, Mesbahi A. Angiosomes of the foot and angiosome-dependent healing. In: Diabetic Foot, Lower Extremity Arterial Disease and Limb Salvage. Philadelphia, PA: Lippincott Williams and Wilkins; 2006.

14. Attinger CE, Cooper P, Blume P. Vascular anatomy of the foot and ankle. Op Tech Plast Reconstr Surg. 1997;4:183-198.

15. Neville RF, Attinger CE, Bulan EJ, et al. Revascularization of a specific angiosome for limb salvage: Does the target artery matter? Ann Vasc Surg. 2009;23:367-373.

16. Setacci C, De Donato G, Setacci F, et al. Ischemic foot: Definition, etiology and angiosome concept. J Cardiovasc Surg (Torino). 2010;51:223-231.

17. Alexandrescu V, Hubermont G, Philips Y, et al. Selective angioplasty following an angiosome model of reperfusion in the treatment of Wagner 1-4 diabetic foot lesions: Practice in a multidisciplinary diabetic limb service. J Endovasc Ther. 2008;15:580-593. 
18. Iida $\mathrm{O}$, Nanto $\mathrm{S}$, Uematsu $\mathrm{M}$, et al. Importance of the angiosome concept for endovascular therapy in patients with critical limb ischemia. Catheter Cardiovasc Interv. 2010;75:830-836.

19. Varela C, Aci NF, Haro JD, et al. The role of foot collateral vessels on ulcer healing and limb salvage after successful endovascular and surgical distal procedures according to an angiosome model. Vasc Endovasc Surg. 2010;44:654-660.

20. Boulton AJM, Armstrong DG. The diabetic foot. In: Clinical Diabetes, Translating Research into Practice. Philadelphia, PA: Saunders Elsevier; 2006.

21. O'Neal LW. Surgical pathology of the foot and clinicopathologic correlations. In: Levin and O'Neal's The Diabetic Foot. Philadelphia, PA: Mosby Elsevier; 2008.

22. Clemens MW, Attinger CE. Angiosomes and wound care in the diabetic foot. Foot Ankle Clin. 2010;15:439-464.

23. Zhu YQ, Zhao JG, Liu F, et al. Subintimal angioplasty for below-theankle arterial occlusions in diabetic patients with chronic critical limb ischemia. J Endovasc Ther. 2009;16:604-612.

24. Bazan HA. Think of the angiosome concept when revascularizing the patient with critical limb ischemia. Catheter Cardiovasc Interv. 2010;75:837.

25. Irvin CL, Guzman RJ. Matrix metalloproteinases in medial arterial calcification: Potential mechanisms and actions. Vascular. 2009;17 Suppl 1: S40-S44.

26. Alexandrescu V. Below-the-ankle subintimal angioplasty: How far can we push this application for lower limb preservation in diabetic patients? J Endovasc Ther. 2009;16:617-618.

27. Alexandrescu V, Ngongang C, Vincent G, Ledent G, Hubermont G. Deep calf veins arterialization for inferior limb preservation in diabetic patients with extended ischaemic wounds, unfit for direct arterial reconstruction: Preliminary results according to an angiosome model of perfusion. Cardiovasc Revasc Med. 2011;12:10-19.
28. Norgren L, Hiatt WR, Dormandy JA, et al. The next 10 years in the management of peripheral artery disease: Perspectives from the PAD 2009 conference. Eur J Vasc Endovasc Surg. 2010;40:375-380.

29. Edmonds M. A natural history and framework for managing diabetic foot ulcers. Br J Nurs. 2008;17:S24-S29.

30. Lyden SP, Smouse HB. TASC II and the endovascular management of infrainguinal disease. J Endovasc Ther. 2009;16 Suppl II:5-18.

31. Ihnat DM, Mills JL. Current assessment of endovascular therapy for infrainguinal arterial occlusive disease in patients with diabetes. J Vasc Surg. 2010;52:92S-95S.

32. Alexandrescu V, Vincent G, Azdad K, et al. A reliable approach to diabetic neuroischemic foot wounds: Below-the-knee angiosome-oriented angioplasty. J Endovasc Ther. 2011;18:376-387.

33. O’Brien-Irr MS, Dosluoglu HH, Harris L, et al. Outcomes after endovascular intervention for chronic critical limb ischemia. J Vasc Surg. 2011;53:1575-1581.

34. Mustapha JA, Heaney CM. A new approach to diagnosing and treating CLI. Endovasc Today. 2010;9:41-50.

35. Apelqvist J, Elgzyri T, Larsson J, et al. Factors related to outcome of neuroischemic/ischemic foot ulcer in diabetic patients. J Vasc Surg. 2011;53:1582-1588.

36. Radford K, Chipchase S, Jeffcoate W. Education in the management of the foot in diabètes. In: The Foot in Diabetes. 4th ed. Chichester, UK: J Wiley and Sons Ltd; 2007.

\section{Publish your work in this journal}

Diabetes, Metabolic Syndrome and Obesity: Targets and Therapy is an international, peer-reviewed open-access journal committed to the rapid publication of the latest laboratory and clinical findings in the fields of diabetes, metabolic syndrome and obesity research. Original research, review, case reports, hypothesis formation, expert opinion and commentaries are all considered for publication. The manuscript management system is completely online and includes a very quick and fair peer-review system, which is all easy to use. Visit http://www.dovepress.com/testimonials.php to read real quotes from published authors. 